\title{
Lossy Mode Resonance Generation by Graphene Oxide Coatings onto Cladding-Removed Multimode Optical Fiber
}

\author{
Miguel Hernaez, Andrew G. Mayes and Sonia Melendi-Espina*
}

\begin{abstract}
In this work, we have studied the suitability of graphene oxide -based thin films to be not only excellent sensitive coatings but also lossy mode resonance (LMR) -generating materials. Thin films of graphene oxide (GO) and polyethylenimine (PEI) fabricated by means of layer-by-layer assembly were selected in this study. Two optical fiber devices with 8 and 20 bilayers of the LMR-generating coating were fabricated and characterized as refractometers. Both devices show no hysteresis and high sensitivity, improving previously reported values. This research opens very promising and exciting possibilities in the field of optical fiber sensors based on LMR, strategically including specific recognition groups to the device surface to exploit this high sensitivity for monitoring a range of target analytes. The carboxylate functional groups at the edges of the GO sheets should provide excellent attachment sites for the required coupling chemistry to realize such devices.
\end{abstract}

Index Terms

Dip-assisted layer-by-layer, graphene oxide, lossy mode resonance, optical fiber sensor, refractometer, thin films

\section{Introduction}

Optical fiber resonance-based sensing devices have attracted the attention of the scientific community in recent years due to their versatility, high sensitivity and robustness [1], [2].

Among the different existing configurations of optical fiber sensors, those based on electromagnetic resonances are the most popular ones. Devices based on Surface Plasmon Resonances (SPR) have become a well-known standard in the field of optical fiber sensors due to the extremely high sensitivities they can reach [1]. However, this family of sensors presents some important drawbacks that limit their use in commercial applications. For example, fewspecific and costly materials, such as gold or silver, are able to generate these resonances and therefore more complicated deposition techniques are necessary [1]. Moreover, the sensing setup required for SPR-based sensors involves the use of complex equipment to control the optical polarization, as this parameter has a dramatic influence in the generation of SPR [1].

Conversely, in the particular case of the emergent Lossy Mode Resonance (LMR)-based sensors, a wide range of materials can support this type of resonance, such as metal oxides and polymers, making these devices more versatile and cheaper [2], [3], [4]. Furthermore, LMR can be excited with both TE and TM polarized light, which crucially simplifies the interrogation scheme [2], [3]. Additionally, a single LMR-based device can produce a multiple-peak response enabling multiple measurements and making the sensors response more robust [3]. Consequently, LMR-based optical fiber sensors can be a promising approach for some applications. 
When an optical waveguide is coated with a thin-film, the propagation of light is affected. Depending on the properties of the materialsinvolved in the system (the waveguide, the coating and the external medium), different cases of electromagnetic resonances can be distinguished [1], [2]. If the real part of the thin-film permittivity is positive and higher in magnitude than both its own imaginary part and the permittivity of the material surrounding the thin film, a LMR is generated [2], [5]. These LMRs produce an absorption band in the transmitted spe ctrum at a determined wavelength. A shift in this resonance peak can be observed when the optical conditions of the system change, that is, when there is a variation in the refractive index (RI) of the coating or the surrounding medium. Consequently, if a thin-film sensitive to a target is deposited onto a waveguide and used as resonance-supporting coating, a variation in this target will produce a measurable shift of the LMR peak [6], [7], [8].

As previously mentioned, LMRs enlarge the range of materials available for sensor surface coating from noble metals, as in the case of SPR, to metal oxides, polymers and many others [3], [9], [10], [11], [12], [13], [14]. In this regard, graphene has inspired significant interest in sensing of various chemical species due to its superior properties such as thermo-electric conduction, surface area and mechanical strength [15], [16], [17], [18].

Graphene is a monolayer of hexagonally arrayed sp2-bonded carbon atoms, extremely sensitive to the external environment. Thus, graphene-based nanostructures have vast potential for developing sensor devices [19] and, consequently, are excellent candidates for the fabrication of the supporting coating required by LMR-sensors. However, the development and applicability of graphene-based sensors is strongly hampered due to the lack of effective methods for scalable graphene production. Fortunately, its precursor material, graphene oxide (GO), can be cheaply mass produced by the chemical exfoliation of inexpensive graphite powder [20]. GO is described as a graphene sheet interrupted by oxygen surface groups in the form of carbonyl, hydroxyl and epoxy groups with carboxylic groups located at the edges [21], [22]. In addition to being easier to produce than pristine graphene, the oxygen-containing functional groups of GO give hydrophilicity, which can be very important, as it enables its dispersion into some solvents for film de position [23], [24], crucial for LMR sensors.

Several authors have explored the potential of GO in different types of optical fiber sensors [19], [25]. However, very few works have investigated the ability of GO as sensitive coating in LMR sensors [26], [27], [28]. In these works, the LMR-based optical fiber sensing scheme consisted of a multimode optical fiber with a layer of $\mathrm{SnO} 2$ directly sputtered onto the core, which performed as LMR-generating coating, and a GO-based thin film deposited on top, acting as sensitive coating. In those studies, very good sensitivities to different targets were achieved, demonstrat ing the suitable properties of $\mathrm{GO}$ to act as an excellent sensitive material.

In our current study, we aim to demonstrate not only the appropriateness of GO as an exceptional sensitive coating, but also its suitability to generate LMRs. This approach simpli fies the fabrication of the devices, as it is possible to avoid the sputtering of a metal oxide layer onto the fiber, required in previous works to generate the LMR. In addition, the present work opens exciting possibilities in the field of optical fiber sensors based on LMR, as the carboxylate functional groups at the edges of the GO sheets should provide excellent attachment sites for the required coupling chemistry to monitor a wide variety of analytes.

Two devices were fabricated and characterized as refractometers, improving the sensitivity achieved by other LMR-generating materials in similar schemes previously reported. To the best of our knowledge, this is the first time that a GO-based thin film is used as LMR-generating coating in optical fiber sensors. 


\section{Materials and methods}

\subsection{Materials and Fabrication of the Sensitive Layer}

To fabricate the sensors, a $200 \mu \mathrm{m}$-core multimode optical fiber (FT200EMT, Thorlabs, Inc.) was used and its cladding was thermally removed. Afterward and prior to the deposition of the LMR-generating layer, the core was sonicated and washed in detergent and acetone and rinsed sufficiently with ultrapure water.

The LMR-generating coating consisted of PEI-GO cross-linked films. GO and PEI were assembled on an interchangeable layer-by-layer basis, by depositing alternating layers of both materials with wash steps in between [26], [27], [28]. The GO powder used was purchased from Graphenea (Spain). The uncladded optical fibers were first hydroxylated by immersion in $1 \mathrm{M} \mathrm{KOH}$ (Sigma Aldrich, UK) for 30 minutes to instigate a negative charge in the fibers followed by rinsing and drying. The layer-by layerassembly commenced with an immersion for 5 minutes in a positively charged $2.0 \mathrm{mg} / \mathrm{mL}$ polyethyleneimine ( $\mathrm{PEI}$, product number 03880, Sigma Aldrich, UK). The positivelycharged fibers were then rinsed in ultra-pure water and dried before immersion for 5 minutes in the negatively charged $0.5 \mathrm{mg} / \mathrm{mL}$ GO suspension. GO nanosheet separation was enhanced by a 30 minute pre -assembly sonication (Ultrawave limited CardiffCF $21 \mathrm{YY}, \mathrm{Hz}-(50-60)$ ). The final LMR-generating coatings of the two fabricated sensors were obtained by repeating these steps 8 and 20 times respectively.

\subsection{Characterization of the LMR-Generating Layer}

To study the LMR-generating coatings in more detail, 8 and 20 bilayers of PEI-GO were also deposited onto silicon-based substrates, following the same assembly procedure previously explained, to examine their quality and thickness. The uniformity and texture of the films were investigated by means of an FEI NanoSEM 450 FEG scanning electron microscope (SEM) and the thickness was measured using a profiling system (DektakXT, Bruker). Ten measurements were taken per sample, calculating the final thickness as the average of all of them.

\subsection{Sensors Characterization}

Two fragments (at around $3 \mathrm{~cm}$ each) of the $200 \mu \mathrm{m}$-core multimode optical fiber were cleaved (NorthLab ProCleave LD II cleaver) and spliced (Fitel S178A fusion splicer) to $200 \mu \mathrm{m}$ optical fiber core pigtails and connected to the characterization setup (Figure 1). This setup consisted of a halogen white light source (HL2000, Oceanoptics Inc.) as the excitation source, connected at one end of the fiber while the other end was connected to a spectrometer (Oceanoptics USB2000), which collected the spectra of the transmitted light through the sensor.

The deposition of the sensitive layer onto the core was carried out foll owing the stepsstated in section II.A. Dip assisted layer by layer was carried out on the pre-assembled devices, characterizing the response after each layer and before depositing the next one. The generation and movement of the LMR absorption bands during the deposition of the LMR-generating layer were monitored using the configuration in Figure 1.

Light was launched into the system from the white light source (Oceanoptics HL2000) and, having passed through the sensitive device, its intensity spectrum was collected by the spectrometer (Oceanoptics USB2000) and shown on the PC screen using OceanView (Oceanoptics), the software developed by the spectrometer manufacturer to control the operation of the device and the capture of the spectra. A reference spectrum (I0) was saved before starting the coating construction. After the deposition of each bilayer of PEI/GO, the optical signal (I) was collected, once the sensor was dry in 
air. OceanView automatically calculated and showed in real time the transmission spectra using the following expression:

$$
T(d B)=10 \log _{10}\left(\frac{I}{I_{0}}\right)
$$

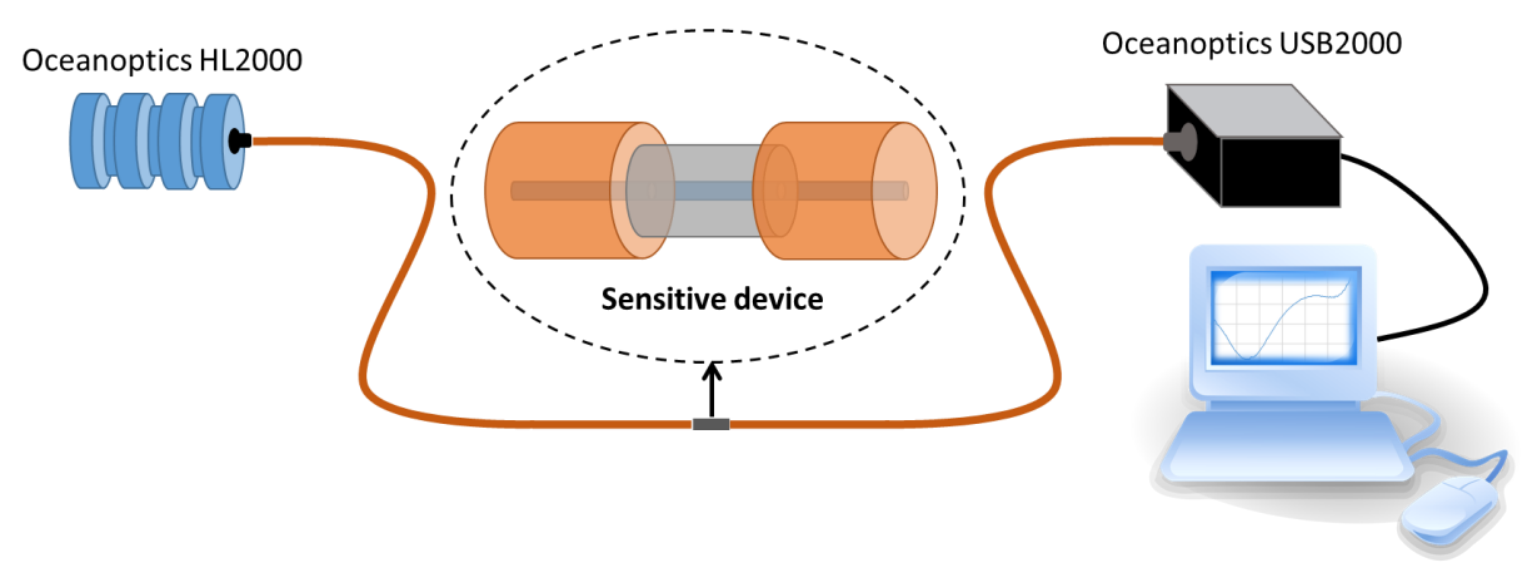

Fig. 1. Sensors characterization setup.

Once both sensors were fabricated, their static and dynamic responses as refractometers were characterized. With this aim, a batch of glycerol-water solutions with glycerol concentrations of $0 \%$, $20 \%, 40 \%$ and $60 \% \mathrm{v} / \mathrm{v}$ were prepared. These concentrations correspond to $\mathrm{RI}$ values at room temperature of 1.333, 1.362, 1.392 and 1.420, respectively [29], [30].

The first experiment consisted in the static characterization of the sensors as refractometers. In this case, the sensor was sequentially immersed in the different glycerol solutions. Initially, the transmission spectrum when the sensor was dry in air was captured and saved. Then, the sensor was immersed into the first glycerol solution. After waiting few seconds to allow the signal to stabilize, the transmission spectrum was collected and observed on the PC screen. Subsequently, the device was withdrawn, thoroughly rinsed with water and dried in air until the captured transmission spectrum moved back to its initial position. This procedure was repeated with all the glycerol solutions, firstly in RI-increasing order and, successively, in RI-decreasing order.

A MatLab routine was created to consistently approximate the obtained absorption band by a polynomial function of degree 2, minimizing the difference between the real peak and the calculated function. With this approach, the results were optimized and it was possible to track shifts of $1 \mathrm{~nm}$ of the LMR absorption bands.

The dynamic performance of the sensors was also studied by sequentiallyimmersing and withdrawing them into two alternating solutions of $40 \%$ and $60 \%$ of glycerol in water every 30 seconds. The transmission spectra were automaticallycaptured every $250 \mathrm{~ms}$ using OceanView. These spectra were post-processed using the above-mentioned MatLab routine to obtain an accurate estimation of the shift of the LMR absorption peaks during the experiment. 


\section{Results}

\subsection{Monitoring of the Generation and Shift of the LMR Absorption Peaks}

As previously mentioned, the deposition of the LMR-generating layer was monitored to follow the generation and shift of the different absorption bands associated with this resonance. These peaks initially appeared in the UV-visible region and moved to the infrared region as the thickness of the deposited PEI-GO thin film increased.

Fig. 2 shows the transmission spectra recorded in air during the construction of the devices. After depositing 4 bilayers of PEI-GO, it is possible to observe the appearance of the first LMR absorption band at $400 \mathrm{~nm}$. This first LMR peak can be seen at $600 \mathrm{~nm}$ with 6 bilayers of the selected coating, while the second LMR band is positioned at $530 \mathrm{~nm}$ once 8 bilayers have been deposited. It was decided to stop the deposition of one of the devices at this point because the position of this peak indicates that the coating thickness is sufficient to make the sensor suitable for further examination.

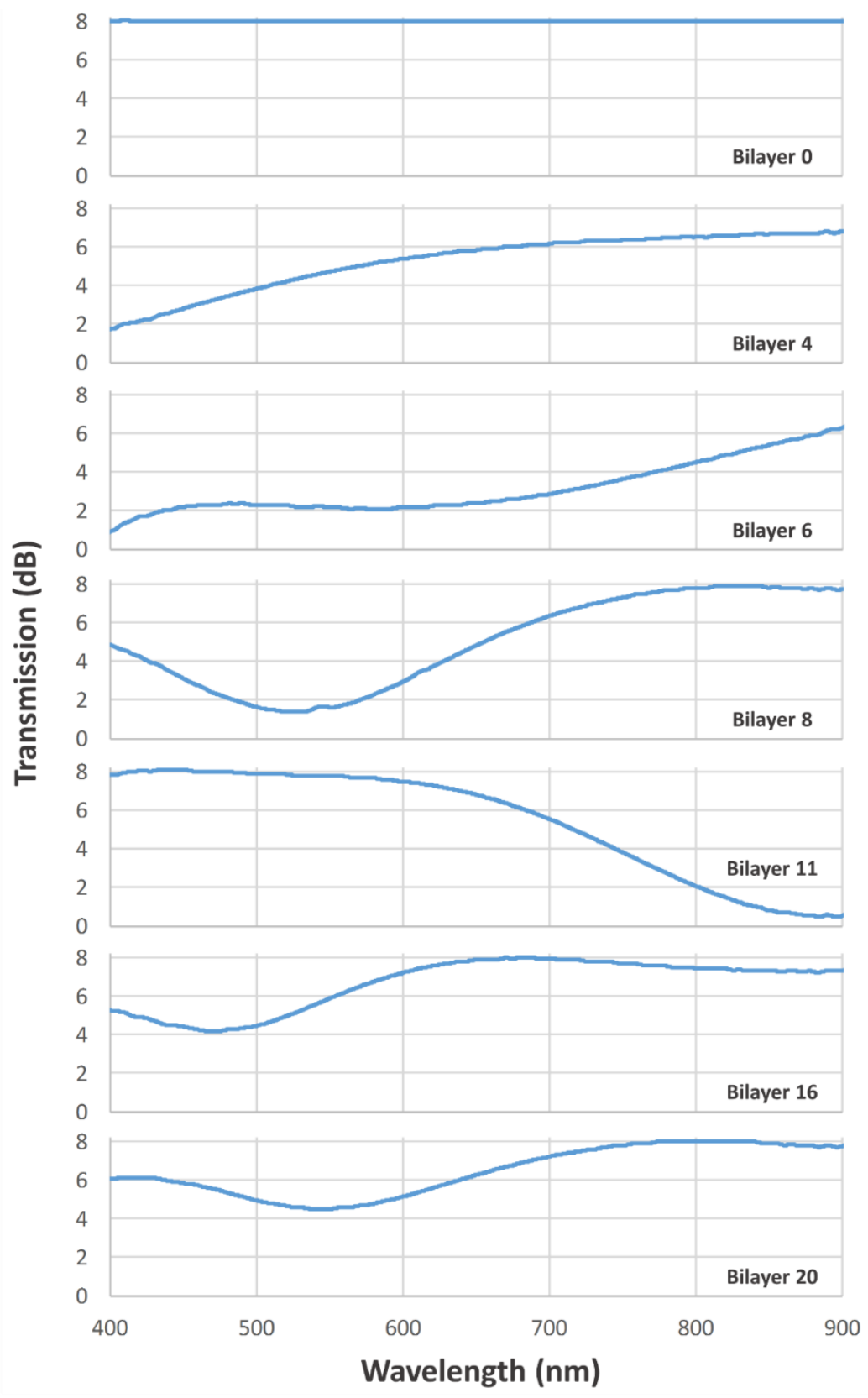

Fig. 2. Transmitted spectra in air during the construction of the LMR-generating coating. 
On the second optical fiber the deposition was resumed up to 20 bilayers. Fig. 2 shows the movement of the second LMR peak from $530 \mathrm{~nm}$ to $898 \mathrm{~nm}$, which is instigated by 11 bilayers of coating. The third LMR absorption band appears at $472 \mathrm{~nm}$, after depositing 16 bilayers of PEI-GO and it shifts to $546 \mathrm{~nm}$ with 20 bilayers.

\subsection{Characterization of the LMR-Generating Layer}

As discussed in the previous section, two devices with 8 and 20 bilayers of the LMR-generating coating were selected for further characterization as refractometers. To study the texture, morphology and thickness of these coatings, 8 and 20 bilayers of PEI-GO were also deposited onto silicon-based substrates. The deposited thin films are uniform and homogeneous (Fig. 3) with average thicknesses of $102 \mathrm{~nm}$ and $330 \mathrm{~nm}$ and standard deviation of $11.7 \mathrm{~nm}$ and $30.0 \mathrm{~nm}$ for the 8 and 20 bilayers, respectively. In Fig. 3 , it is also possible to see the thin films in more detail, where the typical wrinkles characteristic of graphene materials can clearly be observed.
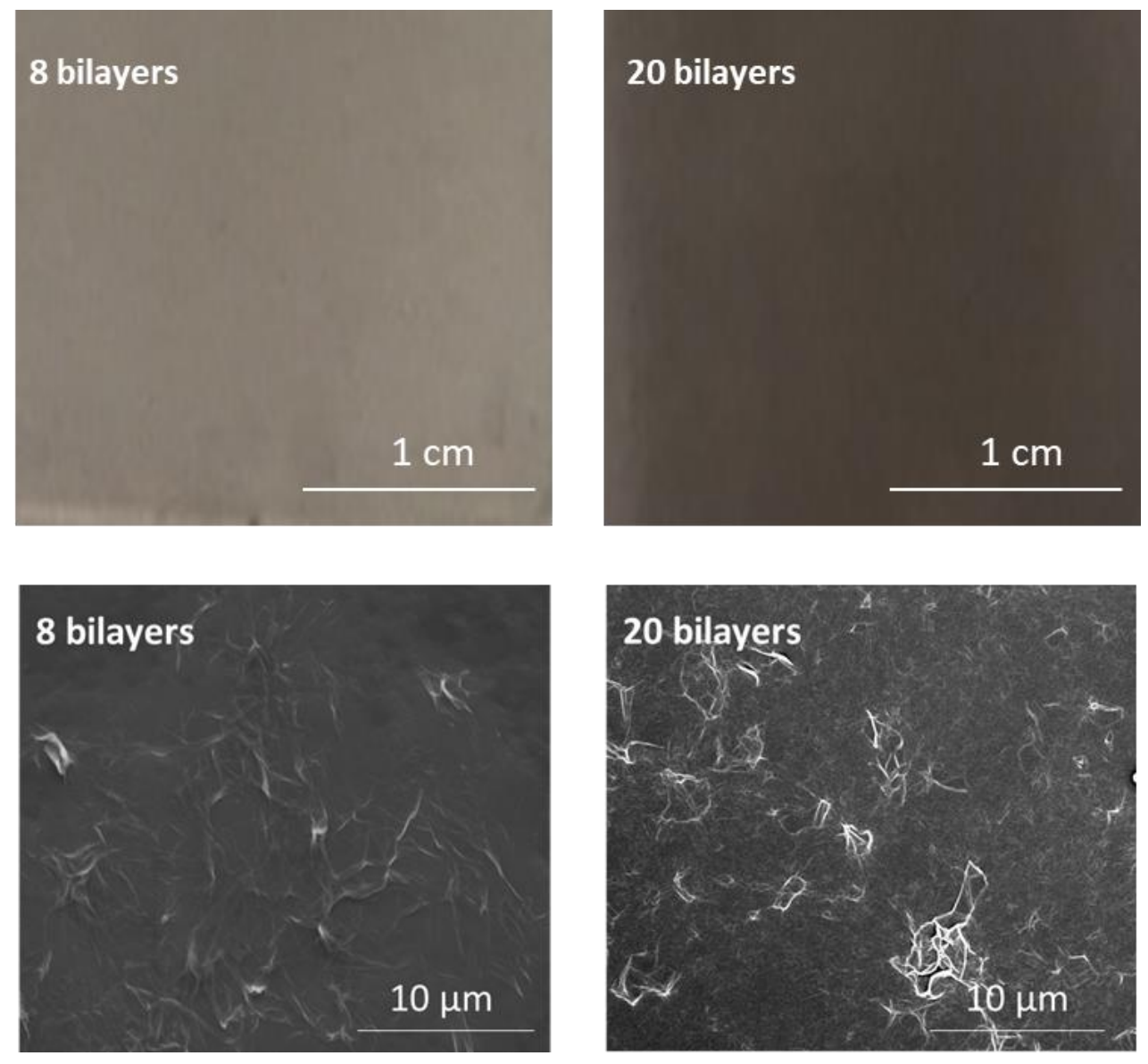

Fig. 3. Top: Pictures of the coatings deposited onto silicon-based substrates. Bottom: SEM images of the coatings deposited onto silicon-based substrates.

\subsection{Characterization of the Devices as Refractometers}

The fabricated optical fiber devices have been statically characterized by immersing them in glycerolwater solutions with different RI and capturing the generated transmitted spectra. Fig. 4 shows the shift of the LMR peaks from the 8 and 20 bilayer devices. 


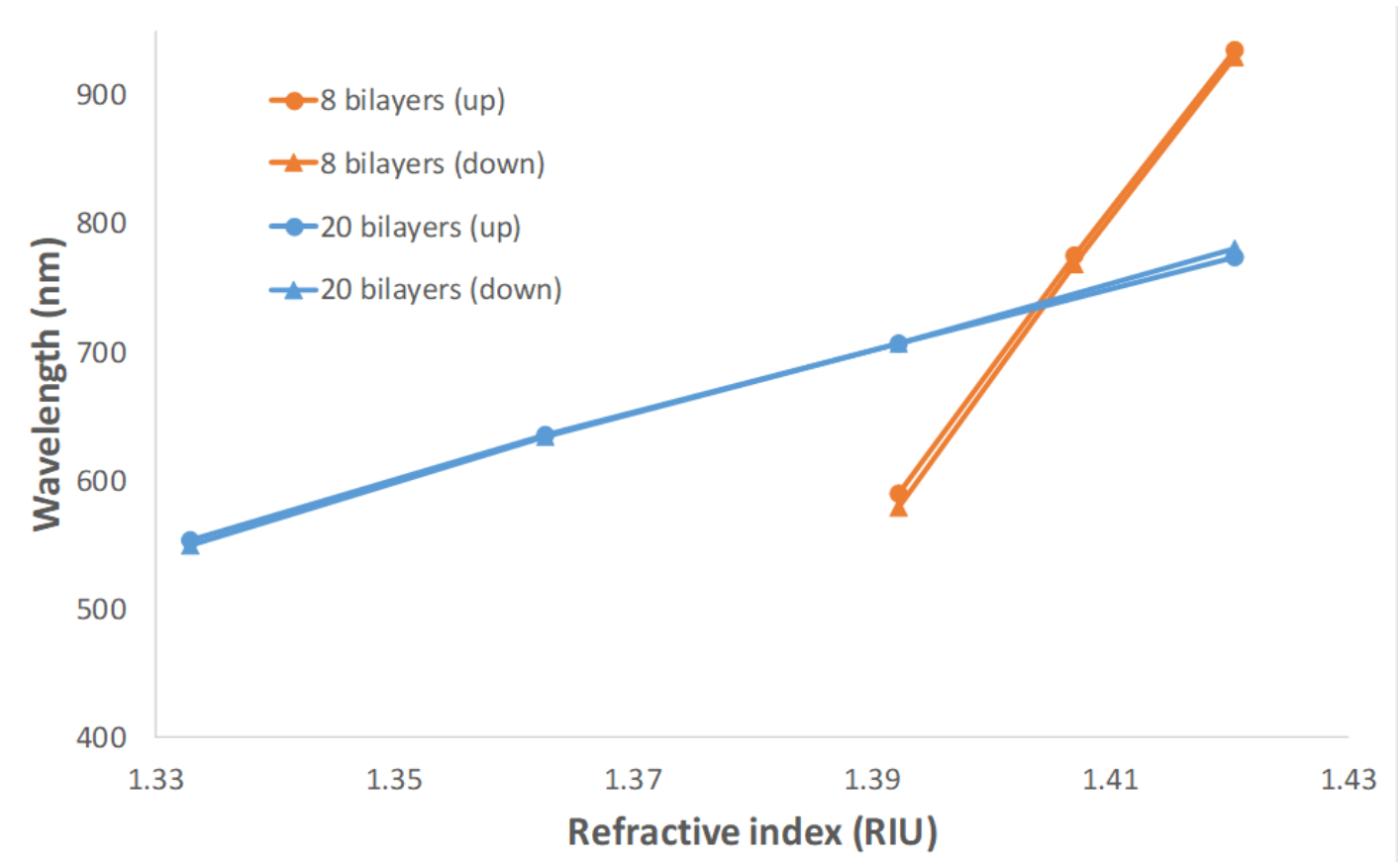

Fig. 4. Wavelength shift of the LMR absorption bands generated by both sensors when they are immersed in solutions with different $R I$.

Both devices exhibit very good reversibility under RI variations. Their responses to increasing and decreasing RI practically overlap, which demonstrates that they display almost no hysteresis in their responses. LMR absorption bands are highly sensitive to variations in the RI of the surrounding medium, especially in the case of the 8 bilayers refractometer.

This device experiences a shift from $590 \mathrm{~nm}$ to $935 \mathrm{~nm}$ with increasing RI and from $930 \mathrm{~nm}$ to $579 \mathrm{~nm}$ with decreasing RI. These values correspond to refractive indices of 1.420 and 1.392 . When this sensor is immersed in solutions with RI of 1.333 and 1.362, the LMR peak falls beyond the scope of the spectrometer, which suggests an outstanding sensitivity over the whole range of measurement. For this reason, there are no wavelength values for those concentrations in the graph. However, to confirm the linear trend, it was decided to submerge this device in a $50 \% \mathrm{v} / \mathrm{v}$ glycerol -water solution with RI 1.407, obtaining a very consistent trend.

The sensitivities for the 8 bilayers device obtained in this range are $12247 \mathrm{~nm} / \mathrm{RIU}$ and $12460 \mathrm{~nm} / \mathrm{RIU}$ for increasing and decreasing refractive indices, respectively. These values improve significantly the sensitivity reached by refractometers based on LMR with coatings of TiO2 [3], ITO [31], In2O3 [32] or even the high sensitivity achieved by a thin-film of sputtered $\mathrm{SnO}_{2}$ [33]. In that study, the maximum sensitivity reported was $5390 \mathrm{~nm} / \mathrm{RIU}$, less than half the value reported here. The estimated resolution of our sensor is $8 \times 10-5 \mathrm{RIU}$ or $0.08 \%$ of glycerol concentration.

The second device with 20 bilayers of the LMR-generating coating exhibits good sensitivity, although it is lower than the previous one. This is consistent with previous studies where the sensitivity decreases when the order of LMR increases [33]. The 20 bilayers refractometer achieves sensitivity values of $2517 \mathrm{~nm} / \mathrm{RIU}$ (immersing it into solutions with RI from 1.333 to 1.420) and $2631 \mathrm{~nm} / \mathrm{RIU}$ into descending RI dissolutions. Thesevalues correspond to an estimated resolution of $3.8 \times 10-4 \mathrm{RIU}$ or, in terms of glycerol concentration, $0.27 \%$. 
The linearity of both devices ( 8 and 20 bilayers) is excellent with correlation factors of $R 2=0.9998$ and 0.9992 respectively.

Both refractometers were also dynamically characterized. The performance of the devices was monitored and captured while they were introduced alternately into solutions of $40 \%$ and $60 \%$ of glycerol in water. Fig. 5 shows the observed wavelength shift versus time.

Both devices respond well and show good repeatability. They completely recover and exhibit very good baseline stability. The response and recovery times were estimated as the required periods to go from $10 \%$ of the wavelength shift and achieve $90 \%$ of maximum signal change and vice versa. The 8 bilayers device is very fast with an average reaction and retrieval time of $1.56 \mathrm{~s}$ and $1.36 \mathrm{~s}$, respectively, while the rise time for the 20 bilayers refractometer is $3.20 \mathrm{~s}$ and it requires an average of $3.81 \mathrm{~s}$ to recover due to the slower diffusion rate though the thicker coating [6].

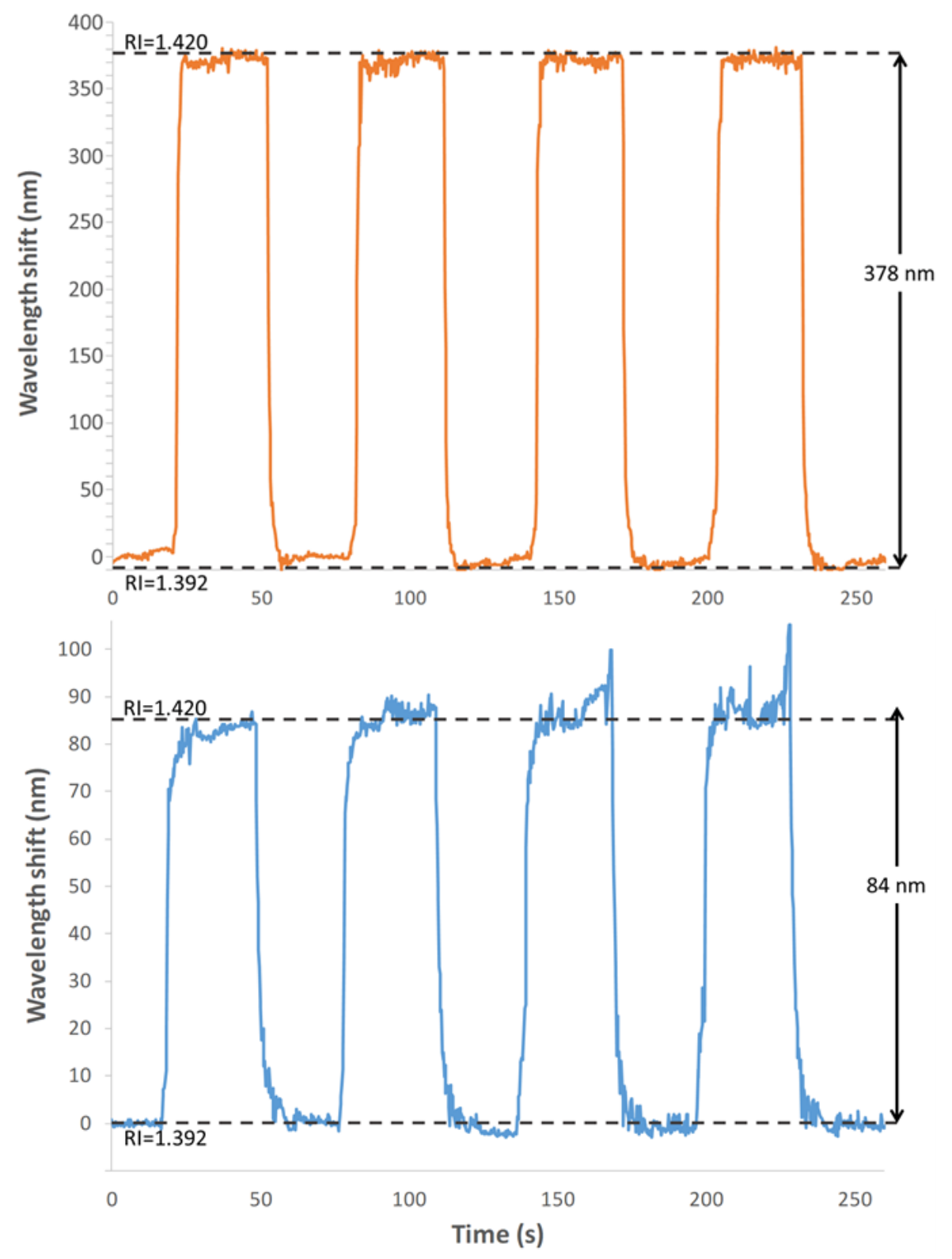

Fig. 5. Dynamic response of both sensors when they are alternatively immersed in solutions with RI of 1.392 and 1.420 . 


\section{Conclusion}

In summary, it is possible to fabricate rapid, stable and very sensitive LMR optical fiber refractometers where GO-based thin films act as sensitive and as LMR-generating coatings. Two optical fiber devices with 8 and 20 bilayers of PEI-GO were made and statically and dynamically characterized as refractometers. LMR bands are very sensitive to changes in the RI of the surrounding media, moving from the UV-visible to the infrared region when the RI increases. The fabricated refractometers show no hysteresis and sensitivity enhancement in comparison with other materials studied in the literature.

To the extent of the authors' knowledge, this is the first work that studies the suitability of GO-based thin films as LMR-generating coatings and the very high sensitivities achieved offer exciting opportunities to add new analyte-selective functionality to the device surface to exploit this high sensitivity for monitoring a range of target analytes. The carboxylate functional groups at the edges of the GO sheets should provide excellent attachment sites for the required coupling chemistry to realize such devices.

\section{Acknowledgment}

Special thanks to our colleagues from UPNA Sensors Research Group (Universidad Pública de Navarra, Spain) who lent us some of their facilities and provided sup port to operate them.

\section{References}

[1] A. K. Sharma, R. Jha, and B. D. Gupta, "Fiber-optic sensors based on surface plasmon resonance: A comprehensive review," leee Sensors Journal, vol. 7, pp. 1118-1129, Jul-Aug 2007.

[2] I. Del Villar, F. J. Arregui, C. R. Zamarreno, J. M. Corres, C. Bariain, J. Goicoechea, C. Elosua, M. Hernaez, P. J. Rivero, A. B. Socorro, A. Urrutia, P. Sanchez, P. Zubiate, D. Lopez, N. De Acha, J. Ascorbe and I. R. Matias, "Optical sensors based on lossy-mode resonances," Sensors and Actuators B-Chemical, vol. 240, pp. 174-185, Mar 2017.

[3] I. Del Villar, M. Hernaez, C. R. Zamarreno, P. Sanchez, C. Fernandez-Valdivielso, F. J. Arregui, et al., "Design rules for lossy mode resonance based sensors," Applied Optics, vol. 51, pp. 4298-4307, Jul 12012.

[4] F. J. Arregui, I. Del Villar, C. R. Zamarreno, P. Zubiate, and I. R. Matias, "Giant sensitivity of optical fiber sensors by means of lossy mode resonance," Sensors and Actuators B-Chemical, vol. 232, pp. 660-665, Sep 2016.

[5] F. Yang and J. R. Sambles, "Determination of the optical permittivity and thickness of absorbing films using long range modes," Journal of Modern Optics, vol. 44, pp. 1155-1163, Jun 1997.

[6] C. R. Zamarreno, M. Hernaez, I. Del Villar, I. R. Matias, and F. J. Arregui, "Optical fiber pH sensor based on lossy-mode resonances by means of thin polymeric coatings," Sensors and Actuators B-Chemical, vol. 155, pp. 290-297, Jul 52011.

[7] M. Hernaez, I. Del Villar, C. R. Zamarreno, F. J. Arregui, and I. R. Matias, "Optical fiber refractometers based on lossy mode resonances supported by TiO2 coatings," Applied Optics, vol. 49, pp. 3980-3985, Jul 102010.

[8] F. J. Arregui, I. R. Matias, J. M. Corres, I. Del Villar, J. Goicoechea, C. R. Zamarreno, et al., "Optical fiber sensors based on Layer-by-Layer nanostructured films," Eurosensors Xxiv Conference, vol. 5, pp. 1087-1090, 2010. 
[9] F. J. Arregui, I. Del Villar, J. M. Corres, J. Goicoechea, C. R. Zamarreno, C. Elosua, et al., "FiberOptic Lossy Mode Resonance Sensors," 28th European Conference on Solid-State Transducers (Eurosensors 2014), vol. 87, pp. 3-8, 2014.

[10] A. B. Socorro, I. Del Villar, J. M. Corres, F. J. Arregui, and I. R. Matias, "Tapered Single-Mode Optical Fiber pH Sensor Based on Lossy Mode Resonances Generated by a Polymeric ThinFilm," leee Sensors Journal, vol. 12, pp. 2598-2603, Aug 2012.

[11] C. R. Zamarreno, S. Lopez, M. Hernaez, I. Del Villar, I. R. Matias, and F. J. Arregui, "Resonancebased refractometric response of cladding-removed optical fibers with sputtered indium tin oxide coatings," Sensors and Actuators B-Chemical, vol. 175, pp. 106-110, Dec 2012.

[12] C. R. Zamarreno, P. Sanchez, M. Hernaez, I. Del Villar, C. Fernandez-Valdivielso, I. R. Matias, et al., "Sensing Properties of Indium Oxide Coated Optical Fiber Devices Based on Lossy Mode Resonances," leee Sensors Journal, vol. 12, pp. 151-155, Jan 2012.

[13] S. P. Usha and B. D. Gupta, "Urinary p-cresol diagnosis using nanocomposite of ZnO/MoS2 and molecular imprinted polymer on optical fiber based lossy mode resonance sensor," Biosensors \& Bioelectronics, vol. 101, pp. 135-145, Mar 152018.

[14] B. D. Gupta, S. P. Usha, and A. M. Shrivastav, "A Novel Approach of LMR/MIP for Optical Fiber based Salivary Cortisol Sensor," 2016 Conference on Lasers and Electro-Optics (Cleo), 2016.

[15] A. H. Castro Neto, F. Guinea, N. M. R. Peres, K. S. Novoselov, and A. K. Geim, "The electronic properties of graphene," Reviews of Modern Physics, vol. 81, pp. 109-162, Jan-Mar 2009.

[16] I. W. Frank, D. M. Tanenbaum, A. M. Van derZande, and P. L. McEuen, "Mechanical properties of suspended graphene sheets," Journal of Vacuum Science \& Technology B, vol. 25, pp. 25582561, Nov 2007.

[17] F. Bonaccorso, Z. Sun, T. Hasan, and A. C. Ferrari, "Graphene photonics and optoelectronics," Nature Photonics, vol. 4, pp. 611-622, Sep 2010.

[18] J. C. Meyer, A. K. Geim, M. I. Katsnelson, K. S. Novoselov, T. J. Booth, and S. Roth, "The structure of suspended graphene sheets," Nature, vol. 446, pp. 60-63, Mar 12007.

[19] M. Hernaez, C. R. Zamarreno, S. Melendi-Espina, L. R. Bird, A. G. Mayes, and F. J. Arregui, "Optical Fibre Sensors Using Graphene-Based Materials: A Review," Sensors, vol. 17, 155, Jan 2017.

[20] W. S. Hummers and R. E. Offeman, "Preparation of Graphitic Oxide," Journal of the American Chemical Society, vol.80, pp. 1339-1339, 1958.

[21] H. Y. He, J. Klinowski, M. Forster, and A. Lerf, "A new structural model for graphite oxide," Chemical Physics Letters, vol. 287, pp. 53-56, Apr 241998.

[22] A. Lerf, H. Y. He, M. Forster, and J. Klinowski, "Structure of graphite oxide revisited," Joumal of PhysicalChemistry B, vol. 102, pp. 4477-4482, Jun 41998.

[23] P. Sanchez, Kandjou, V., Hernaez, M., Zamarreño, C.R., Arregui, F.J., Mayes, A.G., MelendiEspina, S., "Fabrication and characterisation of graphene oxide films with controlled thickness on silicon based substrates," presented at the Carbon Conference, Melbourne, Australia, 2017, p. 302.

[24] V. Kandjou, Perez-Mas, A.M., Hernaez, M. Acevedo, B., Mayes, A.G., Melendi-Espina, S., "The fabrication of controlled thickness graphene oxide films by means of dip-assisted layer by layer assembly," presented at the Carbon Conference, Madrid, Spain, 2018, paper 0649.

[25] Y. Zhao, X. G. Li, X. Zhou, and Y. N. Zhang, "Review on the graphene based optical fiber chemical and biological sensors," Sensors and Actuators B-Chemical, vol. 231, pp. 324-340, Aug 2016.

[26] M. Hernaez, Mayes, A.G., Melendi-Espina, S., "Sensitivity enhancement of lossy mode resonance-based ethanol sensors by graphene oxide coatings, " presented at the IEEESensors 2017 Proceedings, Glasgow, UK, 2017, doi: 10.1109/ICSENS.2017.8234320.

[27] M. Hernaez, A. G. Mayes, and S. Melendi-Espina, "Graphene Oxide in Lossy Mode ResonanceBased Optical FiberSensors for Ethanol Detection," Sensors, vol. 18, 58, Jan 2018. 
[28] M. Hernaez., B. Acevedo, A. G. Mayes and S. Melendi-Espina, "High-performance optical fiber humidity sensor based on lossy mode resonance using a nanostructured polyethyl enimine and graphene oxide coating," Sensors and Actuators B: Chemical, vol. 286, pp. 408-414, 2019.

[29] P. R. Cooper, "Refractive-Index Measurements of Liquids Used in Conjunction with Optical Fibers," Applied Optics, vol. 22, pp. 3070-3072, 1983.

[30] M. Daimon and A. Masumura, "Measurement of the refractive index of distilled water from the near-infrared region to the ultraviolet region," Applied Optics, vol. 46, pp. 3811-3820, Jun 202007.

[31] I. Del Villar, C. R. Zamarreno, P. Sanchez, M. Hernaez, C. F. Valdivielso, F. J. Arregui, et al., "Generation of lossy mode resonances by deposition of high-refractive-index coatings on uncladded multimode optical fibers," Journal of Optics, vol. 12, Sep 2010.

[32] C. R. Zamarreno, P. Sanchez, M. Hernaez, I. Del Villar, C. Fernandez-Valdivielso, I. R. Matias, et al., "Dual-Peak Resonance-Based Optical Fiber Refractometers, "leee Photonics Technology Letters, vol. 22, pp. 1778-1780, Dec 152010.

[33] P. Sanchez, C. R. Zamarreno, M. Hernaez, I. R. Matias, and F. Arregui, "Optical fiber refractometers based on Lossy Mode Resonances by means of SnO2 sputtered coatings," Sensors and Actuators B-Chemical, vol. 202, pp. 154-159, Oct 2014. 